\title{
Triglyceride Down-regulates Expression of MSR-1 in PMA-induced THP-1 Macrophages
}

\author{
Byung Chul Jung ${ }^{1,2, \S,}$, Sung Hoon Kim ${ }^{2,3, \$ * *}$, Sung-Hun Woo ${ }^{2, * * *}$, \\ Jaewon $\operatorname{Lim}^{2,4, \dagger, * *}$ and Yoon Suk Kim ${ }^{2, \dagger, * *}$ \\ ${ }^{I}$ Department of Nutritional Sciences and Toxicology, University of California, \\ Berkeley, State of California 94720, United States \\ ${ }^{2}$ Department of Biomedical Laboratory Science, College of Health Sciences, \\ Yonsei University, Wonju, Gangwon-do 26493, Korea \\ ${ }^{3}$ Department of Biomedical Laboratory Science, Korea Nazarene University, \\ Cheonan, Chungcheongnam-do 31172, Korea \\ ${ }^{4}$ Department of Biomedical Laboratory Science, College of Medical Sciences, \\ Daegu Haany University, Gyeongsan, Gyeongsangbuk-do 38610, Korea
}

\begin{abstract}
Atherosclerosis is a cardiovascular disease in which plaque builds up inside of an artery and can lead to various complications such as myocardial infarction, stroke, and thrombosis. Recently, hypertriglyceridemia has attracted significant attention as contributors to development of atherosclerosis. However, molecular mechanism of its contribution to atherosclerosis is poorly understood. Here we proposed a potential link between triglyceride (TG) and atherosclerosis. TG treatment promoted downregulation of certain scavenger receptor, macrophage scavenger receptor-1 (MSR-1) in phorbol myristate acetate (PMA)-derived human macrophages. TG treatment caused reduction of MSR-1 mRNA expression in a time- and dose-dependent manner. Using chemical inhibitors, we found that inhibition of signaling pathways associated with PI3K and PLC enhances TG-induced reduction of MSR-1 expression in THP-1 macrophages implying that PI3K and PLC is implicated in the expression of MSR-1 in macrophages. Since MSR-1 is associated with uptake and clearance of atherogenic lipoprotein, oxidized low density lipoprotein (oxi-LDL), our data suggest that increase of oxi-LDL due to TG-mediated reduction of its receptor MSR-1 can promote atherosclerosis.
\end{abstract}

Key Words: Triglyceride, MSR-1, THP-1 macrophages, PI3K, PLC

\section{INTRODUCTION}

Triglyceride (TG) is the most abundant dietary fat com- prised of three fatty acids attached to three-carbon glycerol backbone. This lipid is the major form of the stored fat in the human body and plays a pivotal role in providing energy source, structural membrane lipids, and molecules involved

Received: July 21, 2020 / Revised: August 24, 2020 / Accepted: August 26, 2020

*Post-Doctor, ${ }^{* *}$ Professor, ${ }^{* * *}$ Graduate student.

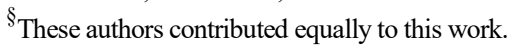

${ }^{\dagger}$ Corresponding author: Yoon Suk Kim. Department of Biomedical Laboratory Science, College of Health Science, Yonsei University, Wonju, Gangwon-do 26493, Korea.

Tel: +82-33-760-2860, Fax: +82-33-760-2195, e-mail: yoonsukkim@yonsei.ac.kr

${ }^{\dagger}$ Corresponding author: Jaewon Lim. Department of Biomedical Laboratory Science, College of Medical Sciences, Daegu Haany University, Gyeongsan, Gyeongsangbuk-do 38610, Korea.

Tel: +82-53-819-1352, Fax:+82-53-819-1353, e-mail: jaewon330@dhu.ac.kr

(C) The Korean Society for Biomedical Laboratory Sciences. All rights reserved.

(c) This is an Open Access article distributed under the terms of the Creative Commons Attribution Non-Commercial License (http://creativecommons.org/licenses/by-nc/3.0/) which permits unrestricted non-commercial use, distribution, and reproduction in any medium, provided the original work is properly cited. 
in signaling pathways (Heier and Kuhnlein, 2018). Since growing body of evidence supports that excess TG levels in blood are associated with an increased risk of atherosclerosis, TG has emerged as a potential pathological factor for atherosclerosis (Menet et al., 2018; Toth et al., 2019). However, the molecular mechanism of its contribution to pathogenesis of atherosclerosis remains to be elucidated.

Macrophages are the major immune cells in atherosclerotic lesions. In arterial plaque, macrophages engulf atherogenic lipoproteins and accumulation of lipid droplets in macrophages leads it to become foam cells, which plays a crucial role in initiating process of atherosclerosis (Ross, 1993; Carmena et al., 2004). In this initial stage of atherosclerosis, atherogenic lipoproteins are internalized by certain macrophage receptor. Scavenger receptor family members SRA1, currently known as macrophage scavenger receptor 1 (MSR1) has been identified as receptors for oxidized low density lipoprotein (oxi-LDL), which is considered as atherogenic lipoprotein (Suzuki et al., 1997; Griffin et al., 2005). Meanwhile, it has been also reported that free atherogenic lipoproteins which were not engulfed by macrophages are risk factors for atherosclerosis (Witztum, 1993; Kolodgie et al., 2000).

In the present study, we show that TG affects expression of MSR-1 in human THP-1-derived macrophages. This report may provide fundamental information for research on the mechanism of development of atherosclerosis.

\section{MATERIALS AND METHODS}

\section{Materials}

PMA was obtained from Sigma-Aldrich (St. Louis, MO, USA). Lipofundin ${ }^{\circledR} \mathrm{MCT} / \mathrm{LCT} 20 \%$ was purchased from B. Braun Melsungen AG (Melsungen, Germany). Lipofundin ${ }^{\circledR}$ MCT/LCT 20\% was used to deliver TG into THP-1 cells as previously described (Aronis et al., 2005). Trypsin-EDTA was purchased from Gibco BRL (Grand Island, NY, USA). TRIzol $^{\circledR}$ for RNA extraction was obtained from Invitrogen (Carlsbad, CA, USA). Chemical inhibitors for JNK (SP600125, $50 \mu \mathrm{M}$ ), p38 MAPK (SB203580, $20 \mu \mathrm{M}$ ), MEK1 (PD98059, $50 \mu \mathrm{M})$, classical PKC (RO31-7549, $0.5 \mu \mathrm{M}$ ), cRAF-1 kinase (GW5074, $0.5 \mu \mathrm{M}$ ), and PI3K (LY294002,
$10 \mu \mathrm{M}$ ) were obtained from Calbiochem (Darmstadt, Germany). A specific inhibitor of NF- $\kappa B$ (BAY11-7085, $1 \mu \mathrm{M}$ ) was obtained from Enzo Life Sciences (New York, NY, USA). A specific inhibitor of PLC (U73122, $50 \mu \mathrm{M}$ ) was purchased from Cayman Chemical (Ann Arbor, MI, USA). Dimethyl sulfoxide (DMSO) was obtained from Sigma-Aldrich (St. Louis, MO, USA). All specific inhibitors were dissolved in DMSO as stock solutions and stored at $-20^{\circ} \mathrm{C}$ prior to use.

\section{Cell culture}

THP-1 human acute monocytic leukemia cell line (ATCC, Manassas, VA, USA) was cultured in RPMI 1640 media supplemented with $10 \%$ fetal bovine serum (FBS) and penicillinstreptomycin and maintained at $37^{\circ} \mathrm{C}$ in a humidified atmosphere with $5 \% \mathrm{CO}_{2}$ (Lee et al., 2017). For differentiation of THP-1 cells into macrophages, THP-1 cells were cultured in 6-well plates $\left(1 \times 10^{6}\right.$ cells/well $)$ and incubated with $200 \mathrm{nM}$ of PMA for $48 \mathrm{~h}$. Thereafter, PMA-derived macrophages were incubated with TG for $24 \mathrm{~h}$ in the presence of chemical inhibitors.

\section{RNA extraction and semi-quantitative reverse transcrip- tase PCR (RT-PCR)}

Total RNA was extracted from THP-1 cells using Trizol ${ }^{\circledR}$ reagent according to the manufacturer's instructions (Yu et al., 2019). cDNA was generated by reverse transcription from $2 \mu \mathrm{g}$ total RNA, $0.25 \mu \mathrm{g}$ of random hexamer (Invitrogen) and 200 units of MMLV-RT (Invitrogen) for $10 \mathrm{~min}$ at $25^{\circ} \mathrm{C}, 50 \mathrm{~min}$ at $37^{\circ} \mathrm{C}$ and $15 \mathrm{~min}$ at $70^{\circ} \mathrm{C}$. cDNA was PCR amplified using Prime Taq premix PCR kit (Genet Bio, Chungnam, Korea) for $20 \sim 40$ cycles using specific primers. Primer sequences are as follows: MSR-1; 5'-CCA GGG ACA TGG GAA TGC AA-3' (forward), 5'-CCA GTG GGA CCT CGA TCT CC-3' (reverse), MARCO; 5'-GTG TCC GTC AGG ATT GTC GG-3' (forward), 5'-ATA TGA GCC CGA GGA CAC CT-3' (reverse). GAPDH was used as an internal control. PCR amplicons were electrophoresed on $2 \%(\mathrm{w} / \mathrm{v})$ agarose gels containing $0.5 \mu \mathrm{g} / \mathrm{mL}$ ethidium bromide and the product size determined by comparison to 100 bp DNA ladder marker (Intron, Gyeonggi, Korea). Gel images were obtained using Gel Doc ${ }^{\mathrm{TM}} \mathrm{XR}+$ system (Bio- 
Rad, Hercules, CA, USA). The PCR product band intensity was measured and normalized against GAPDH using Image $\mathrm{Lab}^{\mathrm{TM}}$ software (version 4.1, Bio-Rad).

\section{Statistical analysis}

$P$-values were calculated using either the Student's $t$-test. Values are shown as mean and standard error of the mean (SEM). Each experiment was conducted three times and the data pooled for analysis.

\section{RESULTS}

MSR-1 mRNA expression is down-regulated in TGtreated THP-1 macrophage

Uptake of oxidized-low density lipoprotein (oxi-LDL)

A

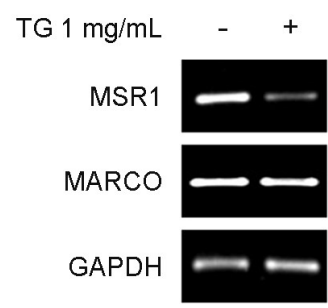

B

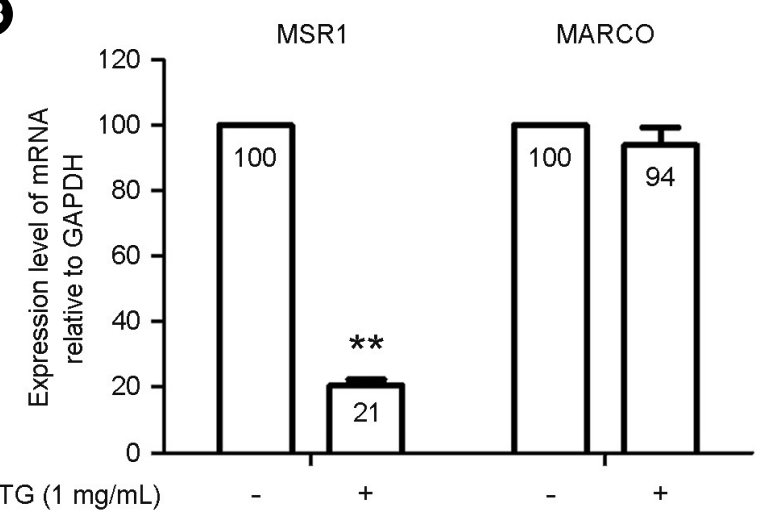

Fig. 1. TG downregulates mRNA level of MSR-1 in PMA-derived THP-1 macrophages. THP-1 macrophages were treated with TG $(1 \mathrm{mg} / \mathrm{mL})$ for $24 \mathrm{~h}$. mRNA levels of MSR-1 and MARCO were analyzed with RT-PCR. The PCR products were electrophoresed on a $2 \%$ agarose gel. (A) A representative image of MSR1 and MARCO expression. (B) Densitometry analysis of PCR products. MSR1 and MSRCO expression level were normalized to GAPDH expression. THP-1 macrophages without TG treatment (control) were set as 100. Data are expressed as the mean \pm SEM from three independent experiments. $P$-values were determined by the Student's $t$-test $(* * P<0.01)$. into macrophages in the atherosclerotic lesion occurs via scavenger receptors including MSR-1 and MARCO. To determine whether TG treatment affects the expression of these scavenger receptor molecules, we treated TG (1 mg/ $\mathrm{mL}$ ) into PMA-induced THP-1 macrophages for $24 \mathrm{~h}$ and analyzed mRNA level of MSR-1 and MARCO through RTPCR. We found that TG treatment of THP-1 macrophages resulted in reduction of MSR-1 expression whereas the expression of MARCO was not significantly changed after TG treatment (Fig. 1). These results indicate that TG treatment reduces mRNA expression of not all but specific type of scavenger receptor, MSR-1, in THP-1 macrophages, which implies TG can potentially inhibit oxi-LDL uptake in macrophages.

\section{TG reduced MSR-1 mRNA expression in THP-1 macro-} phages in a time- and dose-dependent manner

Since TG down-regulated MSR-1 mRNA expression in

A

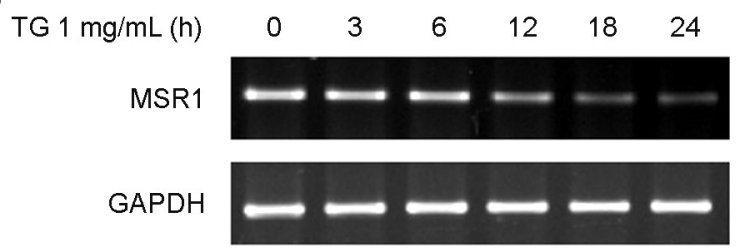

B

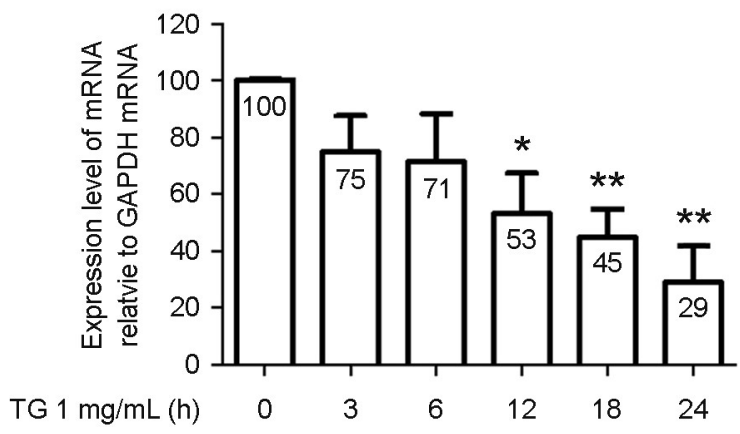

Fig. 2. TG induces decreased expression of MSR-1 mRNA in a time-dependent manner. THP-1 macrophages were cultured with TG $(1 \mathrm{mg} / \mathrm{mL})$ for the indicated times $(0,3,6,12,18$, and $24 \mathrm{~h})$ and MSR-1 mRNA expression was measured by RT-PCR. The PCR products were electrophoresed on a $\%$ agarose gel. (A) A representative image of MSR-1 expression. (B) Densitometry analysis of PCR products. MSR-1 expression levels were normalized to GAPDH expression. Time point $0 \mathrm{~h}$ was set as 100 . Data are expressed as the mean \pm SEM from three independent experiments. $P$-values were determined by the Student's $t$-test $\left({ }^{*} P<0.05,{ }^{* *} P<0.01\right)$. 
(4)

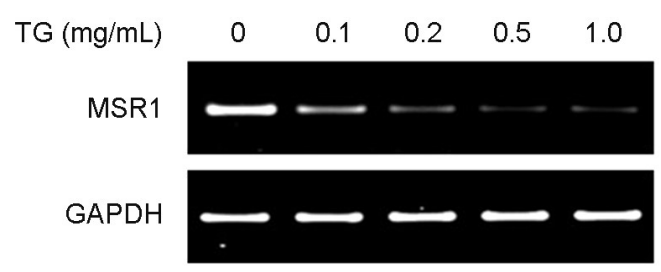

B

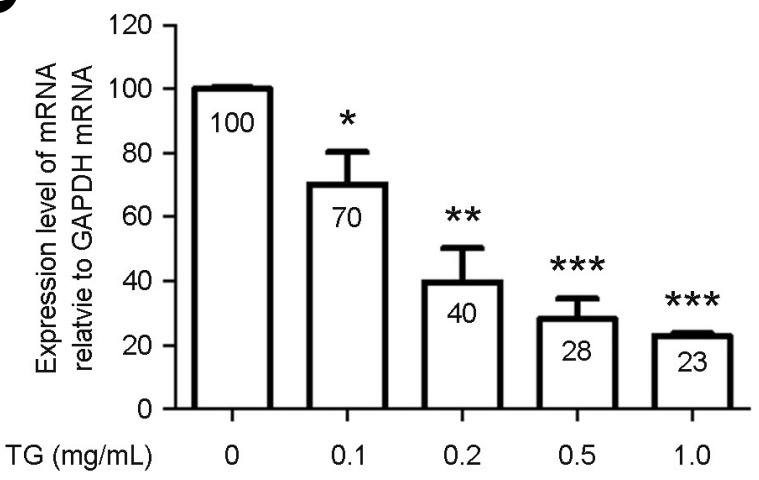

Fig. 3. TG reduced MSR-1 mRNA expression in a dose-dependent manner. THP-1 macrophages were incubated with indicated concentrations of TG $(0,0.1,0.2,0.5$, and $1.0 \mathrm{mg} / \mathrm{mL})$ for $24 \mathrm{~h}$ and MSR-1 mRNA expression assessed by RT-PCR. The PCR products were electrophoresed on a $2 \%$ agarose gel. (A) A representative image of MSR-1 expression. (B) Densitometry analysis of PCR products. MSR-1 expression levels were normalized to GAPDH expression. THP-1 macrophages without TG treatment $(0 \mathrm{mg} / \mathrm{mL})$ were set as 100. Data are expressed as the mean \pm SEM from three independent experiments. $P$-values were determined by the Student's $t$-test $(* P<0.05, * * P<0.01, * * * P<0.001)$.

THP-1 macrophages at $24 \mathrm{~h}$, we further confirmed TGinduced reduction of MSR-1 expression with a time-course experiment. THP-1 macrophages were incubated with TG $(1 \mathrm{mg} / \mathrm{mL})$ for the indicated times $(0,3,6,12,18$, and $24 \mathrm{~h})$ and MSR-1 expression was determined by RT-PCR. Decreased MSR-1 expression reached statistical significance $12 \mathrm{~h}$ after TG treatment and continued to decrease until $24 \mathrm{~h}$ (Fig. 2). We next examined the concentration at which TG could down-regulate MSR-1 mRNA expression. THP-1 macrophages were incubated with TG $(0,0.1,0.2,0.5$, and $1.0 \mathrm{mg} / \mathrm{mL}$ ) for $24 \mathrm{~h}$ and MSR-1 mRNA expression assessed by RT-PCR. We found that $0.1 \mathrm{mg} / \mathrm{mL}$ of TG was sufficient to reduce MSR-1 mRNA expression (Fig. 3). These results indicate that MSR-1 in THP-1 macrophages is downregulated in a time- and dose-dependent manner by TG treatment.
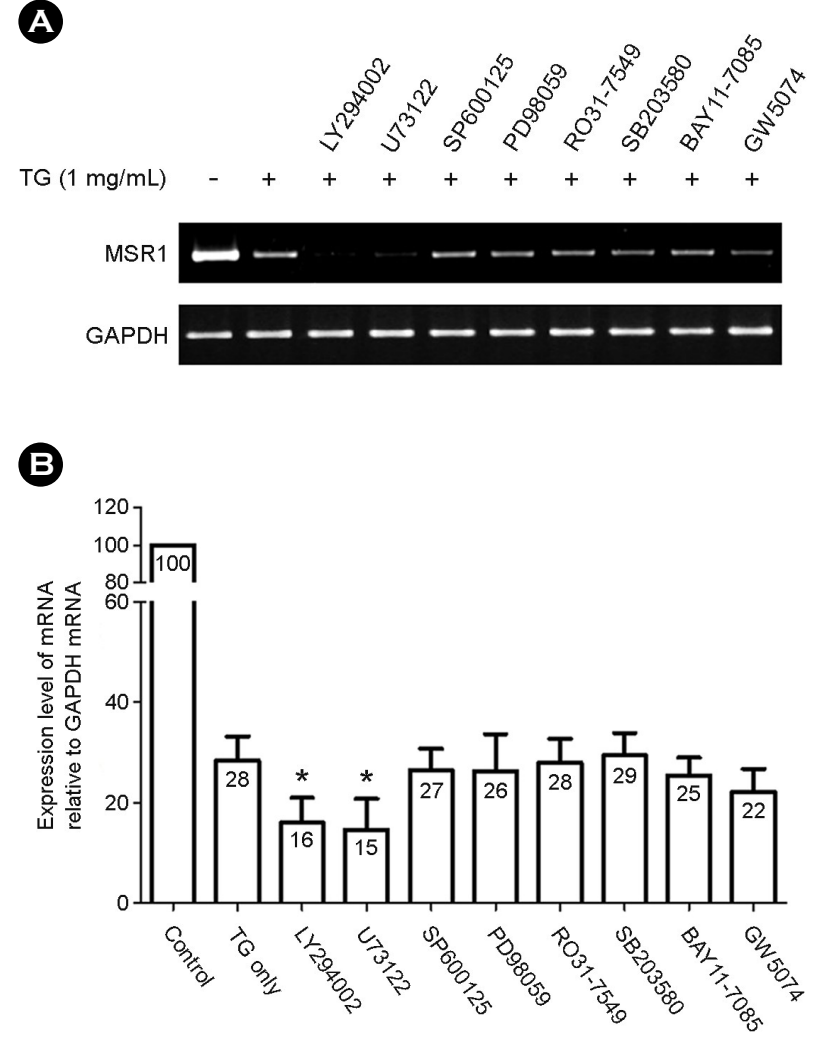

Fig. 4. TG-mediated reduction of MSR-1 expression were strengthened by inhibition of signaling pathways associated with PI $3 \mathrm{~K}$ and PLC. TG $(1 \mathrm{mg} / \mathrm{mL})$ treated THP-1 macrophages were co-cultured with chemical inhibitors for $24 \mathrm{~h}$ and MSR-1 expression assessed by RT-PCR. The inhibitors used are as follows: SP600125 (JNK inhibitor), SB203580 (p38 MAPK inhibitor), PD98059 (MEK1 inhibitor), RO31-7549 (PKC inhibitor), BAY11-7085 (NF-kB inhibitor), LY294002 (PI3K inhibitor), GW5074 (cRAF1 kinase inhibitor), U73122 (PLC inhibitor). The PCR products were electrophoresed on a 2\% agarose gel. (A) A representative image of MSR-1 expression. (B) Densitometry analysis of PCR products. MSR-1 expression levels were normalized to GAPDH expression. THP-1 macrophages without TG treatment $(0 \mathrm{mg} / \mathrm{mL})$ were set as 100 . Data are expressed as the mean \pm SEM from three independent experiments. $P$-values were determined by the Student's $t$-test $(* P<0.05, * * P<$ $0.01, * * * P<0.001)$.

Inhibition of PI3K and PLC enhanced the effect of TG-mediated MSR-1 reduction in THP-1 macrophage

To identify cell signaling pathways involved in downregulation of MSR-1 in TG treated THP-1 macrophages, THP-1 macrophages were treated with TG for $24 \mathrm{~h}$ in the presence of chemical inhibitors. The following signaling molecules were inhibited: PKC, MEK1, NF- $\mathrm{B}, \mathrm{PI} 3 \mathrm{~K}, \mathrm{JNK}$, p38 MAPK, c-Raf, and PLC. After analyzing the expression level of MSR-1, we observed none of the tested chemicals 
rescued TG-mediated reduction of MSR-1. Instead, we found that the groups treated with PI3K inhibitor and PLC inhibitor in the presence of TG further reduced MSR-1 mRNA expression than the groups treated with TG-only (Fig. 4). These results suggest that inhibition of signaling pathways including PI3K and PLC can strengthen the effect of TG on the reduction of MSR-1 expression in THP-1 macrophages, implying that PI3K and PLC is associated with MSR-1 expression in macrophages.

\section{DISCUSSION}

The initial stage of atherosclerosis is highly associated with macrophage transforming into foam cell. When failure of lipid homeostasis, atherogenic lipoproteins in the plasma cross the vascular wall and can be oxidized in vascular subendothelial intima. oxi-LDL, one of the atherogenic modified lipoprotein, is known to promote proinflammatory status, leading to recruitment of monocytes in this region (Kunjathoor et al., 2002). Subsequently, the recruited monocytes are differentiated into macrophages and finally become lipid containing foam cells, occupying much of the atherosclerotic lesion (Rader and Pure, 2005). Therefore, considerable effort has been made to discover a link between foam cell formation and development of atherosclerosis. In this study, we hypothesized TG may affect uptake of oxi-LDL on macrophage via alteration of its receptor molecules. To prove our hypothesis, we treated TG into PMA-derived human THP-1 macrophages. We found that TG down-regulated MSR1 expression in a time- and dose-dependent manner, which may imply that TG involves in the development of atherosclerosis by reducing uptake of oxi-LDL on macrophage via downregulation of its receptor, MSR-1.

Since MSR-1 is associated with development of foam cells, Suzuki and colleagues generated MSR-1 deficiency $\left(\mathrm{MSR}^{-/}\right)$in $\mathrm{ApoE}^{-/-}$mice, the animal model of atherosclerosis (Suzuki et al., 1997). Interestingly, they found reduced size of atherosclerotic lesions in MSR and ApoE double knockout mice, compared with that of $\mathrm{ApoE}^{-/-}$mice. However, this observation was controversial in another well-established mice model for diet-induced atherosclerosis, APOE3Leiden mice. MSR-1 deficiency in this model showed a trend towards increased size of lesion (de Winther et al., 1999). In addition, overexpressed bovine MSR-1 in $\mathrm{Ldlr}^{-/}$mice, another animal model of atherosclerosis, ameliorated a severity of atherosclerotic lesions in aorta (Whitman et al., 2002). Therefore, the outcome of MSR-1 deficiency in atherosclerosis can be dependent on the animal model. Because MSR-1 is highly associated with uptake of atherogenic oxi-LDL, this controversial result can be caused by different oxi-LDL level in respective animal model. Further studies seem to be needed to elucidate how MSR-1 contributes to pathogenesis of atherosclerosis.

In the present study, we demonstrated that challenge of exogenous TG induces down-regulation of MSR1 mRNA level in human macrophage cell line. This result implies excess TG decreased MSR1 on plasma membrane of macrophage, which in turn reduced uptake of oxi-LDL. Subsequently, increased free oxi-LDL activates arterial cells and leads to proinflammatory response, which contributes to recruitment of immune cells in the vessel (Witztum, 1993). Moreover, oxi-LDL can inhibit endothelial derived relaxing factor, which promotes to vasoconstriction, thus lipid deposit can easily build up in the vessel (Chin et al., 1992). All these effects might contribute to development of atherosclerosis. Another important finding in this study is PI3K inhibitor and PLC inhibitor exacerbate the TG effect in terms of MSR1 reduction. This finding brings us one step closer to find the putative molecules, which may recover TG-mediated MSR1 reduction. For example, the putative molecules are likely associated with activation of PI3K and/or PLC pathway. Therefore, our results provide a potential clinical application as well as a molecular mechanism for how TG contributes to development of atherosclerosis.

\section{ACKNOWLEDGEMENT}

This research was supported by a grant from Daegu Haany University Ky-lin Foundation in 2018.

\section{CONFLICT OF INTEREST}

There is no conflict of interest for all authors. 


\section{REFERENCES}

Aronis A, Madar Z, Tirosh O. Mechanism underlying oxidative stress-mediated lipotoxicity: Exposure of j774.2 macrophages to triacylglycerols facilitates mitochondrial reactive oxygen species production and cellular necrosis. Free Radic Biol Med. 2005. 38: 1221-1230.

Carmena R, Duriez P, Fruchart JC. Atherogenic lipoprotein particles in atherosclerosis. Circulation. 2004. 109: III2-7.

Chin JH, Azhar S, Hoffman BB. Inactivation of endothelial derived relaxing factor by oxidized lipoproteins. J Clin Invest. 1992. 89: $10-18$.

de Winther MP, Gijbels MJ, van Dijk KW, van Gorp PJ, suzuki H, Kodama T, Frants RR, Havekes LM, Hofker MH. Scavenger receptor deficiency leads to more complex atherosclerotic lesions in apoe3leiden transgenic mice. Atherosclerosis. 1999. 144: $315-321$.

Griffin EE, Ullery JC, Cox BE, Jerome WG. Aggregated ldl and lipid dispersions induce lysosomal cholesteryl ester accumulation in macrophage foam cells. J Lipid Res. 2005. 46: 2052 -2060 .

Heier C, Kuhnlein RP. Triacylglycerol metabolism in drosophila melanogaster. Genetics. 2018. 210: 1163-1184.

Kolodgie FD, Narula J, Burke AP, Haider N, Farb A, Hui-Liang Y, Smialek J, Virmani R. Localization of apoptotic macrophages at the site of plaque rupture in sudden coronary death. Am J Pathol. 2000. 157: 1259-1268.

Kunjathoor VV, Febbraio M, Podrez EA, Moore KJ, Andersson L, Koehn S, Rhee JS, Silverstein R, Hoff HF, Freeman MW. Scavenger receptors class $\mathrm{a}-\mathrm{i} / \mathrm{ii}$ and $\operatorname{cd} 36$ are the principal receptors responsible for the uptake of modified low density lipoprotein leading to lipid loading in macrophages. J Biol Chem. 2002. 277: 49982-49988.

Lee MH, Kim SH, Ryu SR, Lee P, Moon C. Enhancing the effects of zerumbone on thp-1 cell activation. Korean J Clin Lab Sci.
2017. 49: 1-7.

Menet R, Bernard M, ElAli A. Hyperlipidemia in stroke pathobiology and therapy: Insights and perspectives. Front Physiol. 2018. 9: 488.

Rader DJ, Pure E. Lipoproteins, macrophage function, and atherosclerosis: Beyond the foam cell? Cell Metab. 2005. 1: 223-230.

Ross R. The pathogenesis of atherosclerosis: A perspective for the 1990s. 1993.

Suzuki H, Kurihara Y, Takeya M, Kamada N, Kataoka M, Jishage K, Ueda O, Sakaguchi H, Higashi T, Suzuki T, Takashima Y, Kawabe Y, Cynshi O, Wada Y, Honda M, Kurihara H, Aburatani H, Doi T, Matsumoto A, Azuma S, et al. A role for macrophage scavenger receptors in atherosclerosis and susceptibility to infection. Nature. 1997. 386: 292-296.

Toth PP, Philip S, Hull M, Granowitz C. Association of elevated triglycerides with increased cardiovascular risk and direct costs in statin-treated patients. Mayo Clin Proc. 2019. 94: 1670-1680

Whitman SC, Rateri DL, Szilvassy SJ, Cornicelli JA, Daugherty A. Macrophage-specific expression of class a scavenger receptors in ldl receptor (-/-) mice decreases atherosclerosis and changes spleen morphology. J Lipid Res. 2002. 43: 1201-1208.

Witztum JL. Susceptibility of low-density lipoprotein to oxidative modification. Am J Med. 1993. 94: 347-349.

Yu K, Park S, Chang Y, Hwang D, Kim G, Kim J, Kim S, Kim EJ, Lee D. Evaluation of commercial complementary DNA synthesis kits for detecting human papillomavirus. Korean J Clin Lab Sci. 2019. 51: 309-315.

https://doi.org/10.15616/BSL.2020.26.3.164

Cite this article as: Jung $\mathrm{BC}$, Kim $\mathrm{SH}$, Woo SH, Lim J, Kim YS. Triglyceride Down-regulates Expression of MSR-1 in PMA-induced THP-1 Macrophages. Biomedical Science Letters. 2020. 26: 164-169. 